Mediterránea Ser. Biol. (1989), n. 11. Pág. 121-132

\title{
EL VERDERON COMÚN (Carduelis chloris): POBLACIÓN NIDIFICANTE, TAMAÑO DE LA PUESTA Y ÉXITO REPRODUCTOR. UN EJEMPLO DE ESTUDIOS A LARGO PLAZO
}

\author{
por \\ J.A. GIL-DELGADO ${ }^{1}$, M.C. CATALÁ ${ }^{1}$
}

\section{RESUMEN}

Este artículo presenta algunos aspectos de la estrategia reproductora del verderón común (Carduelis chloris) y los cambios poblacionales durante 14 años de estudio en los naranjales.

La población nidificante muestra un patrón fluctuante con densidades que varían entre 3.5 y 19.5 parejas $\times 10 \mathrm{ha}^{-1}$. La estación de nidificación comienza a finales de marzo y finaliza a principios de agosto. La puesta consta de 2 a 6 huevos y el tamaño medio de la puesta desciende en la segunda mitad de la estación de nidificación. El éxito reproductor varía en el curso de la estación de nidificación y presenta distintos patrones en períodos diferentes. No obstante, la mayor mortalidad coincide con el final de la estación reproductora y tiene su origen en el incremento de las muertes por inanición, la cual se incrementa según avanza la estación de nidificación. La predación no muestra un patrón definido.

\section{SUMMARY}

This study shows the results of an investigation on the breeding biology of the Greenfinch (Carduelis chloris) during 14 breeding seasons (1975-1988) in orange groves located near Sagunto (Valencia, Spain). The breeding season starts during the last days of March and finished in early August (Fig. 1). The clutch-size varies between 2 and 6 eggs, and the average clutch-size decrease in the second half of breeding season. The lower average clutch-size coincides with the end of breeding season (Table II). Breeding success varies along the breeding season and presents different patterns in relation with different periods of time (Table III). The decrease of breeding success as the breeding season proceeds is a consequence of increased starvation. The breeding population varies between 3.5 to 19.5 pairs $\times 10 \mathrm{ha}^{-1}$ (Fig. 2).

Key words: breeding success, clutch-size, starvation, population changes.

(1) Dpto. Ecología, Facultad de Biología, Universidad de Valencia, Burjassot (Valencia). 


\section{INTRODUCCIÓN}

En 1975 comenzamos un estudio sobre las aves que nidifican en los huertos dedicados al cultivo del naranjo. Algunas de las especies que crían en este tipo de cultivos han sido tratadas anteriormente (p.e., Gil-Delgado y Escarré, 1977; Gil-Delgado et al., 1979; Gil-Delgado, 1981; Gil-Delgado y Barba, 1987).

El verderón común (Carduelis chloris) es una especie que en nuestros días está sobre todo asociada a las superficies cultivadas (Lack, 1971; Newton, 1978). Entre éstas, los naranjales representan una fracción importante de los cultivos en el área mediterránea de la Península Ibérica, y el verderón común es una de las especies que nidifican en estos cultivos (Gil-Delgado, 1983).

Wiens (1981 y 1983) critica el valor de los resultados sobre las poblaciones de aves cuando proceden de muestras reducidas. Esta crítica, por extensión, es aplicable a los estudios que se centran en una determinada especie, pues estudios sobre cortos períodos de tiempo únicamente nos permiten vislumbrar lo que existe en ese determinado período (Roth, 1976), además de presuponer situaciones estables que no permiten verificar hipótesis alternativas (véase Wiens, 1983 y 1984).

En este trabajo abordamos los cambios de la población nidificante sobre la misma superficie a lo largo de 14 años de estudio, y algunos aspectos de la biología reproductora del verderón común, tales como el tamaño de la puesta y el éxito reproductor, con dos objetivos básicos: a) explorar los patrones relativos al tamaño de la puesta, éxito reproductor y evolución de la población nidificante durante los 14 años, a los que se ajusta el verderón común en los naranjales, y b) contrastar dichos patrones en relación con la estabilidad o inestabilidad de los mismos.

\section{ÁREA DE ESTUDIO}

El área de estudio está constituida por una parcela dedicada al cultivo del naranjo y que tiene una extensión de 16.919 ha. La parcela está situada en el término municipal de Sagunto; sus características y especies vegetales que la pueblan están descritas en Gil-Delgado y Escarré (1977) y Gil-Delgado et al. (1979). No obstante, la superficie arbolada, que en 1975 cubría la totalidad de la parcela se ha reducido en la actualidad en un $40 \%$, a consecuencia de la renovación de los huertos. Gil-Delgado y Barba (1987) describen el proceso de renovación de los huertos hasta 1986.

\section{MATERIAL Y MÉTODO}

La población nidificante en la parcela se ha determinado mediante la aplicación de la técnica de la parcela (véase García y Purroy, 1973), y de la búsqueda de nidos sobre superficies conocidas (Val Nolan, 1963), método mixto ya empleado sobre otras especies en los naranjales (p.e., Gil-Delgado, 1981). El período de estudio abarca desde 1975 a 1988, aunque la población nidificante no la determinamos con exactitud en 1978 y 1979. Los valores correspondientes a la densidad hacen referencia al período de la temporada repro- 
ductora en que el número de parejas en la parcela es más elevado, con el fin de evitar interferencias a consecuencia del sistema itinerante que muestran los carduelinos durante la estación de nidificación (véase Newton, 1978).

El tamaño de la puesta y el éxito reproductor están basado en una muestra de 119 nidos distribuidos, por años y meses, según muestra la Tabla I. Los 119 nidos cumplen las frecuencias de visitas y las condiciones mínimas señaladas por Gil-Delgado y Escarré (1977) y Gil Delgado et al. (1979). Cada nido está adscrito al mes en el que se depositó el primer huevo. Los que proceden del mes de marzo están agrupados con abril; esta forma de proceder se debe al reducido número de nidos comenzados en marzo (5), y a que en todos ellos el primer huevo procede de los últimos días del mes. Otros 15 nidos localizados en distintas fases de desarrollo, pero que incumplían las condiciones necesarias para determinar el tamaño de la puesta y el éxito reproductor, los hemos utilizado para confeccionar la Figura 1, en cuya parte superior aparecen los intervalos aproximados de todos los nidos pertenecientes a 13 parejas asentadas en 1977 sobre el huerto 118 (véase Figura 1 de Gil-Delgado et al. 1979, para situar este huerto en la parcela).

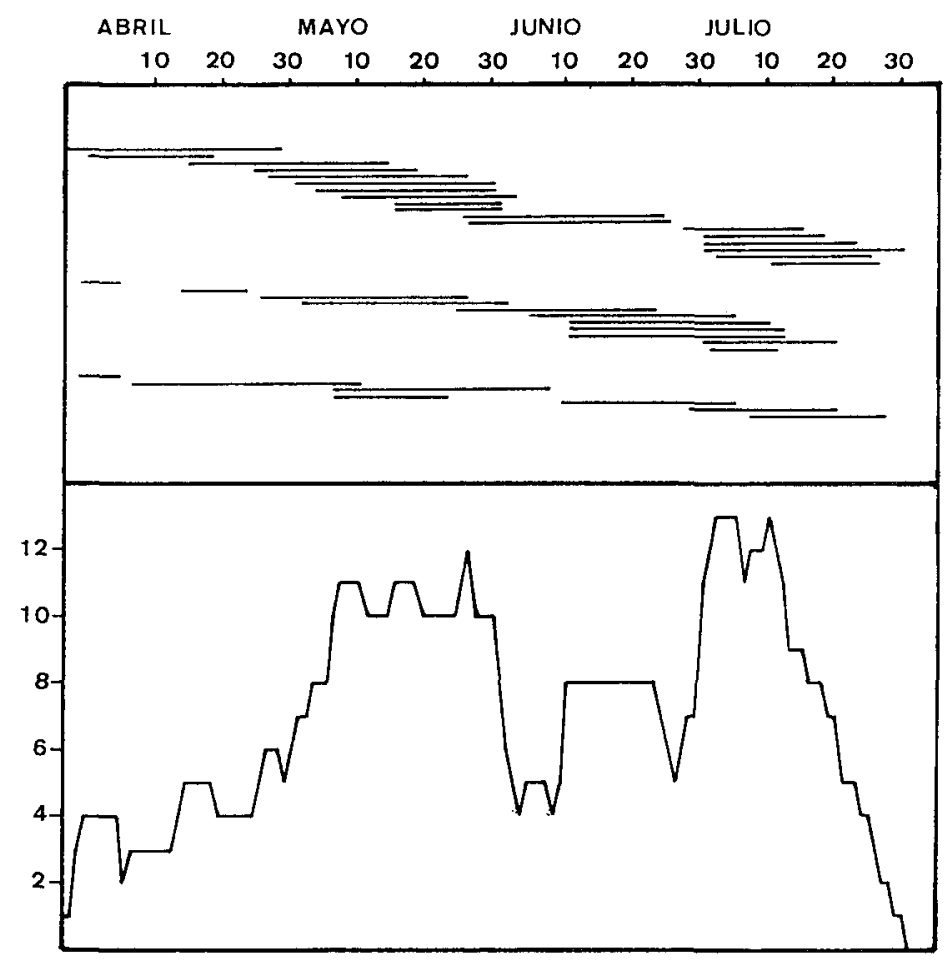

Fígura 1.-Duración de la estación de nidificación del Verderón Común. En la parte superior de la figura se representan todos los nidos (cada línea se corresponde con un nido) de 14 parejas de la temporada reproductora de 1977. En la parte inferior de la figura se muestra el número de nidos activos diarios en la misma temporada reproductora y a partir de los nidos de la parte superior de la figura. 
El tamaño de la puesta lo hemos tratado reuniendo los nidos de todos los años y asignando cada nido al mes del que procede el primer huevo, tras verificar que no existían diferencias significativas entre los mismos meses de los distintos años (Tabla I). Para tratar el éxito reproductor hemos distribuido los nidos en dos períodos (1977-1982 y 1983-1988), a consecuencia del reducido número de nidos en algunos años.

\begin{tabular}{|c|c|c|c|c|c|}
\hline & MARZ0-ABRIL & MAYO & JUNIO & JULLO & TOTAL \\
\hline 1977 & 13 & 19 & 5 & 9 & 46 \\
\hline $1978 \ldots \ldots \ldots \ldots \ldots$ & 4 & 2 & & & 6 \\
\hline $1981 \ldots \ldots$ & 4 & & & & 4 \\
\hline $1982 \ldots \ldots \ldots \ldots \ldots$ & 8 & 6 & 7 & 3 & 23 \\
\hline $1983 \ldots \ldots \ldots \ldots \ldots$ & 6 & 1 & & & 7 \\
\hline $1985 \quad \ldots \ldots \ldots \ldots \ldots$ & 5 & 2 & 6 & & 13 \\
\hline $1986 \ldots \ldots \ldots \ldots \ldots$ & 3 & & & & 3 \\
\hline $1987 \ldots \ldots \ldots \ldots \ldots$ & & 1 & & 3 & 4 \\
\hline $1988 \ldots \ldots \ldots \ldots \ldots$ & 6 & 5 & 2 & & 13 \\
\hline TOTAL ...... & 49 & 36 & 19 & 15 & 119 \\
\hline $\begin{array}{l}F \ldots \ldots \ldots \ldots . . \\
\text { g.l. }\left(n_{1}, n_{2}\right) \ldots\end{array}$ & $\begin{array}{c}1.28 \\
(7,40)\end{array}$ & $\begin{array}{c}1.03 \\
(2,27)\end{array}$ & $\begin{array}{c}2.31 \\
(2,14)\end{array}$ & $\begin{array}{c}0.41 \\
(2,12)\end{array}$ & \\
\hline
\end{tabular}

TABLA I.-Distribución de los 119 nidos por mes y año. Ningún valor de F para contrastar el tamaño de la puesta intramensualmente es significativo. En el ANOVA no se incluyen los meses con 1 y 2 muestras. g.l. = grados de libertad.

\section{RESULTADOS}

La estación de nidificación comienza a finales de marzo y termina en los primeros días de agosto, según podemos apreciar en la Figura 1. La parte superior de la Figura 1 muestra el período de actividad de todos los nidos edificados por 13 parejas que formaban parte de una agregación de 17 sobre el huerto 118. Los nidos de las cuatro parejas que no están incluidos en la figura es consecuencia del asentamiento de las mismas en áreas limítrofes de la parcela, y existen sospechas fundadas de que edificaron nidos en el exterior del área controlada. En 1977 en el huerto 118 se edificaron 41 nidos, 5 de los cuales pertenecían a las cuatro parejas excluidas en la figura; los 36 restantes (parte superior de la Figura 1) muestran que cada pareja edifica 2.8 nidos por término medio (4 parejas, 2 nidos; 8 parejas, 3 ; 1 pareja, 4). La actividad nidificante presenta dos picos, el primero ocupa el mes de mayo y el segundo abarca la primera decena de julio (parte inferior de la Figura 1). Este patrón equivale a dividir la estación de nidificación en dos partes; la primera correspondiente a las nidadas iniciales, con la inserción de puestas de reposición y segundas puestas de la parejas que inician la temporada reproductora más temprano, y una segunda parte que tiene su origen en las segundas y terceras puestas. 


\section{Tamaño de la puesta}

El tamaño de la puesta varía entre 2 y 6 huevos. Los tipos de puesta más frecuentes son las formadas por 4 y 5 huevos (Tabla II). La Tabla II también muestra el tamaño medio de la puesta para cada uno de los meses en que hemos distribuido la estación de nidificación. El tamaño medio de la puesta varía en el curso de la temporada reproductora $\left(\mathrm{F}_{3,14}=5.48 ; \mathrm{p}<0.01\right)$. Las diferencias se explican por el descenso del tamaño medio de la puesta en la segunda mitad de la estación de nidificación, ya que no hay diferencias entre marzo-abril y mayo $\left(F_{1,83}=1.39 ;\right.$ n.s. $)$ ni entre junio y julio $\left(F_{1,32}=0.83 ;\right.$ n.s. $)$.

\begin{tabular}{|c|c|c|c|c|c|c|c|}
\hline PUESTA & & & & & & & \\
\hline MES & 2 & 3 & 4 & 5 & 6 & $\mathbf{x}$ & s.d \\
\hline Marzo-Abril & 1 & 4 & 13 & 29 & 2 & 4.5 & 0.78 \\
\hline Mayo & & 2 & 7 & 25 & 2 & 4.7 & 0.64 \\
\hline Junio & 1 & 2 & 9 & 7 & & 4.1 & 0.8 \\
\hline Julio & 2 & 3 & 5 & 5 & & 3.9 & 1.02 \\
\hline
\end{tabular}

TABLA II.-Tamaño de la puesta en relación con el mes del que proceden los nidos.

\section{Éxito reproductor}

La Tabla III muestra el éxito reproductor del verderón común para cada mes y para cada uno de los dos períodos en que hemos distribuido los nidos. En la misma Tabla se han representado los valores de $\mathrm{X}^{2}$ procedentes de contrastar los mismos meses de los dos períodos $\left(\mathrm{X}_{\mathrm{M}}^{2}\right)$ y los obtenidos de contrastar los distintos meses de cada uno de los períodos considerados $\left(\mathrm{X}_{\mathrm{T}}^{2}\right)$. Los valores obtenidos muestran que en los dos períodos el éxito reproductor varía en el curso de la estación de nidificación. Sin embargo, el mismo tipo de análisis entre los mismos meses únicamente aprecia diferencias en marzo-abril (Tabla III).

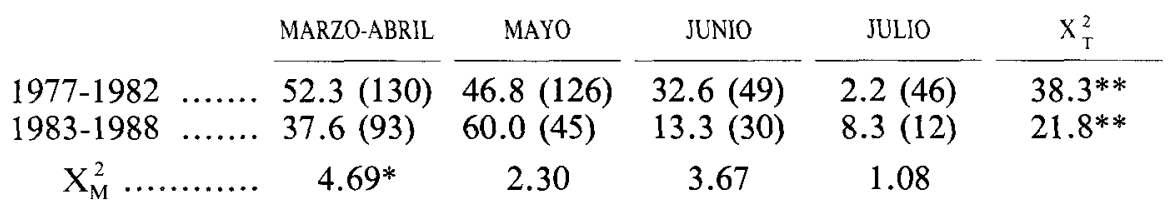

TABLA III.-Éxito reproductor en relación con el mes de iniciación de las nidadas para los dos períodos que hemos considerado. Los valores se ofrecen en porcentajes sobre el número de huevos (cifra entre paréntesis). $\mathrm{X}_{\mathrm{T}}^{2}$ contrasta los distintos meses de cada periodo; $\mathrm{X}_{\mathrm{M}}^{2}$ contrasta los mismos meses de los dos períodos.

$(* \mathrm{p}<0.05 ; * * \mathrm{p}<0.001)$. Grados de libertad respecto a $\mathrm{X}_{\mathrm{T}}^{2}=3 ; y$ respecto a $\mathrm{X}_{\mathrm{M}}^{2}=1$. 
Los dos períodos muestran distinto patrón, pues mientras en el correspondiente a 1977-1982 la supervivencia desciende según avanza la estación de nidificación, en el segundo período (1983-1988), la supervivencia se incrementa desde marzo-abril a mayo $\left(\mathrm{X}^{2}=6.00 ; \mathrm{p}<0.02 ; \mathrm{g} .1 .=1\right)$, para a continuación descender hasta el final de la estación reproductora (véase Tabla III).

Las principales causas de mortalidad de huevos y pollos son la predación y la inanición. En la Tabla IV sintetizamos el efecto que cada una de ellas tiene en el desarrollo de las nidadas, con el fin de explorar cuál de las dos causas es la responsable de la mayor mortalidad durante el período final de la estación de nidificación.

Los resultados (Tabla IV) muestran claramente que las muertes por inanición aumentan según avanza la estación de nidificación $\left(\mathrm{X}^{2}=44.26 ; \mathrm{p}<0.001\right.$; g.1. =3). Por el contrario, la predación no muestra diferencias significativas entre los distintos meses por los que transcurre la temporada reproductora $\left(\mathrm{X}^{2}=5.41 ; \mathrm{p}>0.05 ; \mathrm{g} .1 .=3\right)$.

\begin{tabular}{|c|c|c|c|c|}
\hline & MARZO-ABRIL & MAYO & JUNIO & JULIO \\
\hline Huevos predados ....... & 20 & 15 & & \\
\hline Pollos predados .......... & 39 & 18 & 24 & 11 \\
\hline Predación ..... & 59 & 33 & 24 & 11 \\
\hline$\ldots .$. & 26.4 & 19.3 & 30.4 & 18.9 \\
\hline Inanición ... & 23 & 31 & 22 & 27 \\
\hline$\% \quad \ldots \ldots \ldots$ & 10.3 & 18.1 & 27.8 & 46.5 \\
\hline Otras causas .............. & 38 & 21 & 13 & 18 \\
\hline
\end{tabular}

TABLA IV.--Distribución de las causas de mortalidad en relación con el mes de procedencia de las nidadas. Los porcentajes están en relación con el número de huevos depositados en cada mes, cifra que se puede obtener a partir de los valores que están entre paréntesis en la tabla III.

\section{La población nidificante}

En nuestra área de estudio el verderón común presenta grandes variaciones y se ajusta a un patrón fluctuante (Figura 2). Esta variación es apreciable a partir de esperar pequeñas desviaciones, si la población es estable, respecto a la media de las 12 temporadas reproductoras en que determinamos con exactitud la población nidificante. Sin embargo, las diferencias son altamente significativas $\left(\mathrm{X}^{2}=39.17 ; \mathrm{p}<0.001 ; \mathrm{g} .1 .=11\right)$.

Durante el período de estudio la población presenta dos picos, años 1977 y 1983, y dos valores mínimos en 1981 y 1987 (Figura 2). Aunque desconocemos con exactitud la población nidificante en 1978 y 1979, ambas temporadas mantenían densidades superiores a 1981 (7.1 y 6.5 parejas $\mathrm{x} 10 \mathrm{ha}^{-1}$ son las densidades mínimas para 1978 y 1979, respectivamente). Por otra parte, la elevada densidad de 1977 está supeditada al agregado de 17 parejas establecidas en el huerto $118(53.1 \%$ de las parejas nidificantes $)$. Estas agregaciones no las hemos vuelto a observar en temporadas posteriores, pese a que Newton 


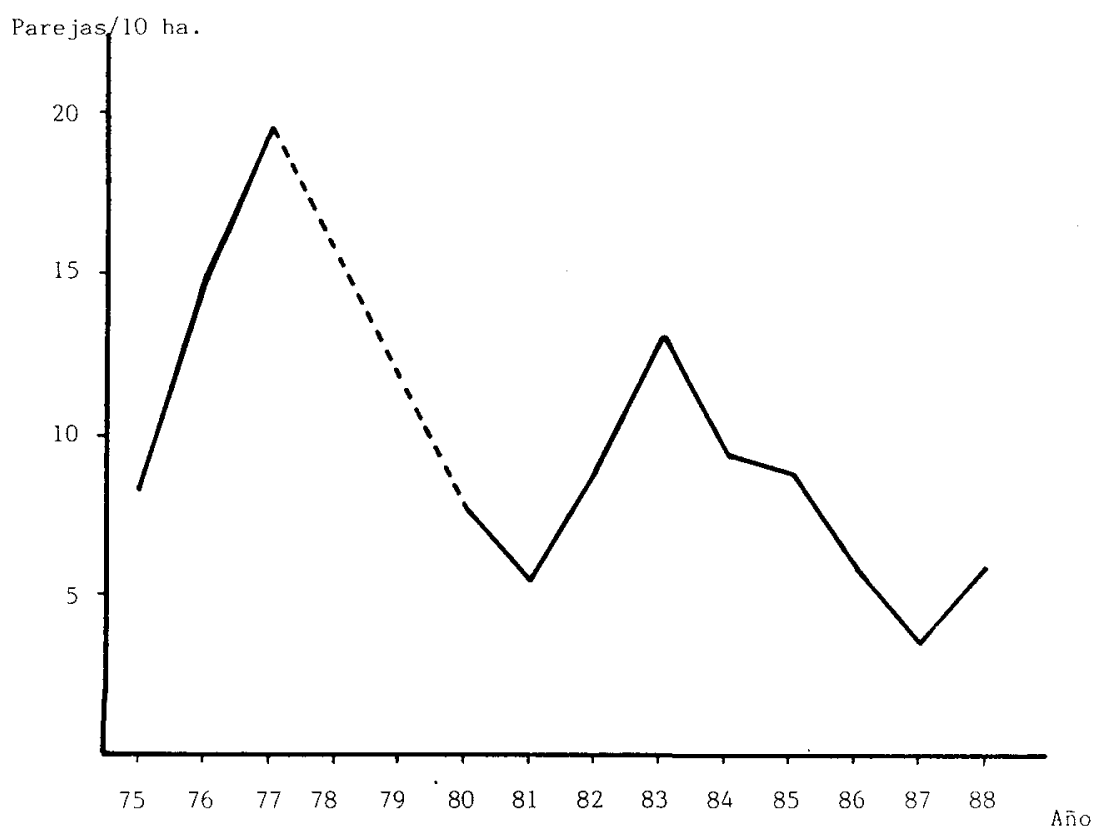

Figura 2.-Población nidificante en los naranjales saguntinos durante el período de estudio. Los resultados se refieren a valores de densidad $\left(n .^{\circ}\right.$ parejas $\times 10 \mathrm{ha}^{-1}$. La línea discontinua se corresponde con los años 1978-79.

(1978) señala que los carduelinos durante la estación de nidificación forman pequeños agregados formados por un reducido número de parejas en donde cada una de ellas defiende un pequeño territorio.

\section{DISCUSIÓN}

Gnielka (1986) señala que el verderón común inicia la estación de nidificación en la DDR en la primera decena del mes de marzo, y es durante abril y mayo cuando la actividad reproductora es más intensa. En Sagunto existe un cierto retraso respecto a la población alemana e incumple la tendencia a comenzar la estación de nidificación con anterioridad según disminuye la latitud (véase Perrins y Birkhead, 1983). Además, en Sagunto se aprecia un segundo pico durante el mes de julio (Figura 1).

En Sagunto el tamaño medio de la puesta se reduce en la segunda mitad de la estación de nidificación, y es al final de la misma cuando las puestas contienen un menor número de huevos (véase Tabla II). Este patrón es diferente al obtenido por Gnielka (1986) en la DDR, donde el menor tamaño de la puesta tiene lugar al principio de la temporada reproductora. En Gran Bretaña el tamaño medio de la puesta es similar durante toda la estación de nidificación (véase apéndice 5 en Newton, 1978). Por otra parte, el tamaño medio de la 
puesta en nuestra área de estudio no experimenta cambios entre los mismos meses de diferentes años.

El éxito reproductor se ajusta a dos patrones: a) aumenta entre marzoabril y mayo para a continuación decrecer hasta el final de la estación de nidificación, y b) disminuye en el curso de la estación de nidificación. Ambos patrones muestran que el verderón común tiene dificultades en criar las polladas al final de la estación de nidificación. Esta situación está determinada por las muertes que ocasiona la inanición, la cual aumenta según avanza la temporada reproductora. Por el contrario, la predación no presenta un patrón definido. Newton (1978) señala que el efecto de la predación disminuye según avanza la estación de nidificación como resultado del aumento de la cobertura vegetal, que origina una mayor protección de los nidos, y consecuentemente, generando una mayor producción de pollos al final de la temporada reproductora. En los naranjales, los efectos derivados de la poda originan una situación inversa según avanza la estación de nidificación, siendo predecible un aumento de la predación si ésta tuviera su origen en la menor cobertura vegetal. No obstante, la predicción no se cumple. Por otra parte, el menor éxito reproductor de las polladas al final de la estación de nidificación también lo muestra el verdecillo (Serinus serinus) en este mismo hábitat (Gil-Delgado, 1981), aunque hasta junio el verderón común presenta una supervivencia superior al de la otra especie mencionada (compárese la Tabla III con la Tabla IV de GilDelgado, 1981).

Lack (1954) sugiere que el tamaño de la puesta concuerda con el del número de pollos que los progenitores pueden criar, y es consecuente con la predicción de que la tendencia estacional en el tamaño medio de la puesta operará en paralelo con el éxito reproductor (Murphy, 1978). Alternativamente, von Haartman (1971) postula que las especies de aves con capacidad de variar el número de huevos dejan tantos huevos como ellas son capaces de depositar. En los naranjales el verderón común y el verdecillo muestran el menor tamaño medio de la puesta al final de la estación de nidificación, en coincidencia con la época en que el éxito reproductor es menor. Desde la perspectiva de Lack (1954), el menor tamaño medio de la puesta durante este período es el preámbulo de las dificultades con las que se van a encontrar para criar sus polladas. El que la predación no muestre un patrón definido a lo largo de la estación de nidificación, y que las diferencias deriven del aumento de la inanición, son consecuentes con la predicción y la hipótesis de Lack. No obstante, el reducido número de crías que consiguen abandonar el nido durante el tramo final de la estación de nidificación contrasta con las esperanzas teóricas de los progenitores. Newton (1986) muestra que un incremento de alimento incide positivamente en el número de huevos depositados. La destrucción del tapiz vegetal durante mayo y junio tiene que influir necesariamente de forma negativa sobre los pollos al disminuir los recursos alimentarios. Esta reducción de recursos debe de incidir también sobre las hembras desde que durante la oogénesis ella debe gastar nutrientes y energía en los huevos (Murphy, 1978), de forma que una disminución de alimento disponible tiene como respuesta la deposición de un menor número de huevos, lo que estaría de acuerdo con la hipótesis de von Haartman, y ninguna de las dos hipótesis puede ser refutada.

El que algunos pollos mueran con los buches repletos (Gil-Delgado, obs. pers.) durante el tramo final de la estación de nidificación, implica que la ca- 
rencia de alimento no explica toda la mortalidad durante este período. Esta situación nos permite plantear: ¿cuál es la pretensión de las últimas puestas si los beneficios obtenidos son practicamente nulos? Nuestros resultados no permiten generar una respuesta, pero la elevada mortalidad al final de la estación reproductora debe de ser consecuencia de eventos no esperados que atentan con fuerza contra la viabilidad de los pollos.

El verderón común en los naranjales presenta un patrón poblacional fluctuante con densidades comprendidas entre las 3.5 y 19.5 parejas $\times 10 \mathrm{ha}^{-1}$. En los hábitats naturales de la Península Ibérica la especie no es detectada como nidificante (Purroy, 1975; Herrera, 1980; Santos y Suárez, 1983, entre otros), o bien, presenta densidades muy bajas (Santos et al., 1983). Nuestros resultados confirman su asociación a las superficies cultivadas (Lack, 1971; Newton, 1978). En áreas antrópicas de Italia, Lo Valvo et al. (1985) determinan densidades comprendidas entre los valores obtenidos en los naranjales. La Figura 2 permite apreciar un período de crecimiento de la población de 2 años al que le siguen 4 años en que la población disminuye. Sin embargo, las densidades, máxima y mínima, correspondientes al período 1982-88 son un tercio inferiores a las densidades, máxima y mínima, del período 1975-81. Esta disminución de la población nidificante en la segunda mitad del período de estudio puede explicarse a partir de la disminución del área arbolada en la parcela (véase GilDelgado y Barba, 1987), desde que el tipo y la estructura del hábitat influyen en la composición de las especies de aves y en las densidades específicas (Johnston y Odum, 1956; Orians, 1971; Murton Westwood, 1974; Welsh 1983; Blondel, 1985; Morse, 1985; Potti, 1985; Hirons y Johnson, 1987, entre otros). No obstante, para el verderón común este argumento puede resultar insuficiente por su tendencia a criar en agregados (Newton, 1978), al tiempo que el proceso de renovación del arbolado supone temporalmente aumentar, al menos teóricamente, la cantidad de recursos alimentarios disponibles por buscar el alimento, en unión de otros carduelinos, en los huertos con plantones o desprovistos de árboles (Gil-Delgado, 1979).

De los tres aspectos tratados con mayor amplitud en este artículo: tamaño de la puesta, éxito reproductor y población; únicamente el primero muestra un patrón similar durante el período de estudio. Los dos restantes muestran fuertes variaciones a lo largo de los 14 años de estudio. Desde que la totalidad de las aves que residen en un determinado hábitat son el resultado de la adición de las distintas especies que están presentes en el hábitat, nuestros resultados abogan por la necesidad de estudios a largo plazo que permitan vislumbrar los patrones a los que se ajustan. Generalizar conclusiones nacidas al amparo de un reducido tamaño muestral en combinación con cortos períodos de tiempo en la obtención de los datos básicos, determina generar respuestas a partir de datos aleatorios que impiden descubrir las verdaderas tendencias y patrones a los que se ajustan las diferentes especies en particular y el conjunto de las mismas (véase Wiens, 1981, 1983 y 1984).

\section{AGRADECIMIENTOS}

Hemos de agradecer a E. Barba y G. López la ayuda prestada. También consideramos las sugerencias de un revisor anónimo que sirvieron para mejorar este artículo. 


\section{BIBLIOGRAFIA}

BLONDEL, J. (1985). Habitat selection in island versus mainland birds. En Habitat selection in birds, 477-516. Cody M.L. (ed). Academic Press.

GARCÍA, L., y PURROY, F. (1973). Evaluación de comunidades de aves por el método de la parcela. Resultados obtenidos en el matorral mediterráneo de la Punta del Sabinar (Almería). Bol. Est. Cent. de Ecología 4: 41-49.

GIL-DELGADO, J. A. (1979). La ornitocenosis de los naranjales (Sagunto, Valencia). Tesis doctoral (no editada). Fac. de Biología, Univ. de Valencia.

GIL-DELGADO, J. A. (1981). La avifauna del naranjal valenciano. III. El Verdecillo (Serinus serinus L.). Mediterränea 5: 97-114.

GIL-DELGADO, J. A. (1983). Breeding bird community in orange groves. Proceedings VII Int. Con. Bird Census IBCC V Meeting EOA, 100-106.

GIL-DELGADO, J. A., y BARBA, E. (1987). Aves nidificantes en huecos de los naranjos. Mediterránea 9: 29-40.

GIL-DELGADO, J. A., y ESCARRÉ, A. (1977). La avifauna del naranjal valenciano. I. Datos preliminares sobre Mirlo (Turdus merula L.). Mediterránea 2: 89-109.

GIL-DELGADO, J. A.; PARDO, R.; BELLOT, J., y LUCAS, I. (1979). Avifauna del naranjal valenciano II. El Gorrión Común (Passer domesticus L.). Mediterránea 3: 69-99.

GNIELKA, R. (1986). Daten zur Brutbiologie des Grünfinken (Carduelis chloris) nach Nestkarten aus dem Berzik Halle. Beitr. Vogelkd. 32: 235-244.

HER RERA, C. M. (1980). Evolución estacional de las comunidades de Paseriformes en dos encinares de Andalucía occidental. Ardeola 25: 143-180.

HIRONS, G., y JONHSON, T. H. (1987). A quantitative analysis of habitat preferences of Woodcock Scolopax rusticola in the breeding season. Ibis 129:371-381.

JOHNSTON, D. W., y ODUM, E. P. (1956). Breeding bird population in relation to plant succession on the piedmont of Georgia. Ecology 37: 51-62.

LACK, D. (1954). The natural regulation of animal number. Oxford University Press.

LACK, D. (1971). Ecological isolation in birds. Blackwell.

LO VALVO, M.; LA MANTIA, T., y MASSA, B. (1985). Bird population of Palermo's urban and suburban areas. Boll. Zool. 52: 347-354.

MORSE, D. H. (1985). Habitat selection in North American Parulid Warblers. En Habitat selection in birds. Cody M.L. (eds.). Academic Press.

MURPHY, E.C. (1978). Seasonal variation in reproductive output of House Sparrows: The determination of clutch size. Ecology 59: 1189-1199.

MURTON, R.K. y WESTWOOD, N.J. (1974). Some effects of agricultural change on the English avifauna. British Bird 67: 41-69.

NEWTON, I. (1978). Finches. Collins.

NEWTON, I. (1986). The Sparrowhawk. T and AD Poyser.

ORIANS, G. (1971). Ecological aspects of Behavior. En Avian Biology I: 513-546. Farner D.S. y King J.R. (eds.). Academic Press.

PERRINS, C.M. y BIRKHEAD, T.R. (1983). Avian Ecology. Blackie.

POTTI, J. (1985). La sucesión de las comunidades de aves en los pinares repoblados de Pinus sylvestris del macizo de Ayllón (Sistema Central). Ardeola 32: 253-277.

PURROY, F. (1985). Evolución anual de la avifauna de un bosque mixto de coníferas y frondosas en Navarra. Ardeola 21: 669-697.

ROTH, R.R. (1976). Spatial heterogeneity and bird species diversity. Ecology 57: 773-782.

SANTOS, T. y SUÁREZ, F. (1983). The bird communities of the heathlands of Palencia. The effects of coniferous plantations. Proceedings VII Int. Con. Bird Census IBCC V Meeting EOAC, 172-179.

SANTOS, T., SUÁREZ, F. y TELLERÍA, J. L. (1983). The bird communities of Iberian Juniper woodlands (Juniperus thurifera L.). Proceeding VII Int. Con. Bird Census IBCC V Meeting EOAC, 79-88.

VAL NOLAN, Jr. (1963). Reproductive success of birds in a deciduous scrub habitat. Ecology 44: 305-313.

VON HAARTMAN, L. (1971). Population dynamics, 391-459. En Avian Biology. D.S. Farner y J.R. King (eds). Academic Press.

WELSH, D.H. (1983). Use of the mapping method to study the effect of boreal forest cutting on bird populations. Proceedings VII Int. Con. Bird Census IBCC V Meeting EOAC, 57-63.

WIENS, J.A. (1981). Single-sample surveys of communities: are the revealed patterns real? Am. Nat. 117: $90-98$. 
WIENS, J.A. (1983). Avian community ecology: an iconoclastic view. En Perspectives in Ecology, 355-403. Brush A.H. y Clark G.A. (eds). Cambridge University Press.

WIENS, J.A. (1984). On understanding a non-equilibrium world: myth and reality in community patterns and processes. En Ecological communities: conceptual issues and the evidence, 439457. Stron D.H., Simberloff D., Abele L.G. y Thistle A.B. (eds). Princenton University Press. 